\title{
Thermally Generated Gauge Singlet Scalars as Self-Interacting Dark Matter
}

\author{
John McDonald* \\ CERN, Theory Division, 1211 Geneva, 23, Switzerland
}

(Received 5 July 2001; revised manuscript received 23 July 2001; published 15 February 2002)

\begin{abstract}
We show that a gauge singlet scalar $S$, with a coupling to the Higgs doublet of the form $\lambda_{S} S^{\dagger} S H^{\dagger} H$ and with the $S$ mass entirely generated by the Higgs expectation value, has a thermally generated relic density $\Omega_{S} \approx 0.3$ if $m_{S} \approx(2.9-10.5)\left(\Omega_{S} / 0.3\right)^{1 / 5}(h / 0.7)^{2 / 5} \mathrm{MeV}$. Remarkably, this is very similar to the range $\left[m_{S}=(6.6-15.4) \eta^{2 / 3} \mathrm{MeV}\right]$ required in order for the self-interaction $(\eta / 4)\left(S^{\dagger} S\right)^{2}$ to account for self-interacting dark matter when $\eta$ is not much smaller than 1 . The corresponding coupling is $\lambda_{S} \approx$ $\left(2.7 \times 10^{-10}-3.6 \times 10^{-9}\right)\left(\Omega_{S} / 0.3\right)^{2 / 5}(h / 0.7)^{4 / 5}$, implying that such scalars are very weakly coupled to the standard model sector.
\end{abstract}

PACS numbers: 95.35. $+\mathrm{d}, 12.60 . \mathrm{Fr}, 98.80 . \mathrm{Cq}$

1. Introduction.-It has become apparent that conventional collisionless cold dark matter (CCDM) may have problems accounting for the observed structure of galaxies. $N$-body simulations with CCDM indicate that galaxies should have singular halos [1,2] with large numbers of subhalos $[3,4]$. Observationally, the density profile of galaxies in the inner few kiloparsecs appears to be much shallower than predicted by numerical simulations (the central density of dark matter halos being 50 times smaller than the CCDM prediction for dwarf galaxies and roughly independent of halo mass [2,5]), while the number of dwarf galaxies in the local group is an order of magnitude fewer than predicted $[3,4]$. In addition, the CCDM predictions for the Tully-Fisher relation [6,7] and the stability of galactic bars in high surface brightness spiral galaxies [8] are not in agreement with what is observed, indicating lower density galaxy cores than predicted by CCDM. Although there is at present considerable uncertainty regarding the interpretation of observations and simulations $[9,10]$, it has nevertheless been argued that all the discrepancies between observations and simulations may be understood as indicating that dark matter halos in CCDM simulations are more centrally concentrated than observed [11].

In order to overcome the possible deficiencies of CCDM halos, one suggestion has been that the cold dark matter particles have a nondissipative self-interaction $[12,13]$, and it has been shown that such cold, nondissipative selfinteracting dark matter (SIDM) can be effective in alleviating the various problems of CCDM [11]. Scattering of dark matter particles stops gravitational accretion at the center of the halo and so allows a smooth core to form. Simulations with SIDM [11,14] are able to simultaneously account for the observed density profiles of galactic halos and the number of subhalos [11]. In the future SIDM may be strongly constrained by gravitational lensing observations of the shape of cluster halos [15-17] and by the formation of massive black holes at the centers of galaxies, which is enhanced by self-interactions of dark matter particles [18].

In order to be able to account for the observed properties of dark matter halos, the requirement on the mass $M$ and self-interaction scattering cross section $\sigma$ of the SIDM particles is that [13]

$$
r_{S}=\frac{\sigma}{M}=\left(2.05 \times 10^{3}-2.57 \times 10^{4}\right) \mathrm{GeV}^{-3} .
$$

The upper bound corresponds to the limit at which galaxy halos in massive clusters are destroyed by interacting with hot particles in the cluster halo (evaporation) [13], while the lower bound corresponds to the limit where the SIDM particle would not interact within a typical galactic halo during a Hubble time $[12,13]$.

The canonically simplest dark matter particle is arguably a gauge singlet scalar $S$. The possibility that gauge singlet scalars, interacting with the standard model sector via a coupling to the Higgs doublet of the form $S^{\dagger} S H^{\dagger} H$, could naturally constitute dark matter has been pointed out by a number of authors in the past $[19,20]$ as well as more recently [21]. These calculations consider the case of massive $(>1 \mathrm{GeV})$ scalars which freeze out of thermal equilibrium when nonrelativistic [22]. However, the range of $S$ masses considered is too large to account for SIDM with perturbative $S$ couplings.

It has recently been noted that gauge singlet scalars have a natural self-interaction via an $S^{4}$-type coupling and so in principle could account for SIDM [23-25]. An estimate of the upper bound on the coupling of $S$ scalars to the Higgs doublets for $S$ mass of the order of $10-100 \mathrm{MeV}$ (which is of the greatest interest in the case of perturbative $S$ self-interactions) was derived in [24] by requiring that $S$ scalars do not come into thermal equilibrium and so overpopulate the Universe.

In this Letter we consider thermal generation of SIDM $S$ scalars which do not achieve equilibrium. We will show that such nonequilibrium thermal generation can naturally account for a dark matter density of $S$ scalars with the right properties to account for SIDM.

The Letter is organized as follows. In Section 2 we discuss the perturbative upper limit on the $S$ scalar mass. In Section 3 we consider the thermal generation of a relic density of $S$ scalars. In Section 4 we consider the case of zero bare $S$ mass and the resulting consistency of the relic 
density, $S$ mass, and Spergel-Steinhardt SIDM cross section for natural values of the $S$ self-coupling. In Section 5 we present our conclusions.

2. Limit on $m_{S}$ for perturbative SIDM.-We first consider the perturbative upper limit on the $S$ mass if it is to play the role of SIDM. We will consider the case of complex gauge singlet scalars for consistency with the cross sections and discussion given in [20], which we will use here. We expect that the results for real scalars will be very similar. The model is described by

$$
\mathcal{L}=\partial_{\mu} S^{\dagger} \partial^{\mu} S-m^{2} S^{\dagger} S-\lambda_{S} S^{\dagger} S H^{\dagger} H-\frac{\eta}{4}\left(S^{\dagger} S\right)^{2} .
$$

The total center-of-mass $S$ scattering cross section is the sum of $S S^{\dagger} \rightarrow S S^{\dagger}$ and $S S \rightarrow S S$,

$$
\sigma \equiv \sigma_{S S^{\dagger} \rightarrow S S^{\dagger}}+\sigma_{S S \rightarrow S S}=\frac{3 \eta^{2}}{128 \pi m_{S}^{2}} .
$$

Therefore

$$
r_{S}=\frac{\sigma}{m_{S}}=\frac{3 \eta^{2}}{128 \pi m_{S}^{3}},
$$

which implies that

$$
m_{S}=35.8 \alpha_{\eta}^{1 / 3}\left(\frac{2.05 \times 10^{3} \mathrm{GeV}^{-3}}{r_{S}}\right)^{1 / 3} \mathrm{MeV},
$$

where $\alpha_{\eta}=\eta^{2} / 4 \pi$. (Similar expressions have been obtained in [24,25].) Thus if we require that $\alpha_{\eta} \lesssim 1$ in order to have a perturbative theory, then the condition Eq. (1) requires that $m_{S}=\alpha_{\eta}^{1 / 3}(15.4-35.8) \mathrm{MeV} \lesssim 30 \mathrm{MeV}$. (We refer to this as the Spergel-Steinhardt mass range.) We note that this puts a severe bound on the coupling $\lambda_{S}$, since the $S$ scalar gains a mass from the Higgs expectation value,

$$
m_{S}^{2}=m^{2}+\frac{\lambda_{S} v^{2}}{2}
$$

where $v=250 \mathrm{GeV}$. Thus the requirement that $m_{S} \lesssim$ $30 \mathrm{MeV}$ imposes an upper bound on $\lambda_{S}$,

$$
\lambda_{S}<2.9 \times 10^{-8}\left(\frac{m_{S}}{30 \mathrm{MeV}}\right)^{2} .
$$

The Spergel-Steinhardt mass range assumes a perturbative $S$ self-coupling. A nonperturbative self-coupling may be possible, but it would be difficult to calculate the properties of such a model, so we must restrict ourselves to the perturbative case. In addition, the only known scalar self-coupling, that of the standard model Higgs doublet, $\lambda_{H}\left(H^{\dagger} H\right)^{2}$, is given by $\lambda_{H}=m_{h}^{2} / 4 v^{2}=0.053\left(m_{h} / 115 \mathrm{GeV}\right)^{2}$. (The experimental lower bound on the Higgs mass is $m_{h}>113 \mathrm{GeV}$ [26], while an upper bound for the pure standard model is obtained from radiative corrections to electroweak observables, $m_{h}<165 \mathrm{GeV}$ [27]. The upper bound in extensions of the standard model can be $1 \mathrm{TeV}$ or larger [28].) This is typically perturbative but not very much smaller than 1 , suggesting that a natural value for the $S$ scalar self-couplings is around 0.1 .

2. Thermal generation of $S$ scalars.-There are two processes which can produce a density of $S$ scalars: $2 \leftrightarrow 2$ annihilation processes and decay of a thermal equilibrium density of Higgs scalars to $S S^{\dagger}$ pairs, $h^{o} \rightarrow S S^{\dagger}$. We first consider $2 \leftrightarrow 2$ annihilations. The relic density from scattering processes in a radiation dominated Universe is found by solving the Boltzmann equation [20,22],

$$
\frac{d f}{d T}=\frac{\left\langle\sigma_{\mathrm{ann}} v_{\mathrm{rel}}\right\rangle}{K}\left(f^{2}-f_{0}^{2}\right) ; \quad K=\left[\frac{4 \pi^{3} g(T)}{45 M_{\mathrm{Pl}}^{2}}\right]^{1 / 2},
$$

where $f=n_{S} / T^{3}, f_{0}=n_{0} / T^{3}$, and $g(T)=g_{B}+7 g_{F} /$ 8 , where $g_{B}$ and $g_{F}$ denote the number of relativistic bosonic and fermionic degrees of freedom, respectively. $n_{S}$ is the number density of $S$ scalars and $n_{0}$ is the thermal equilibrium $S$ number density; for relativistic $S$ scalars

$$
n_{0}=\left(\frac{1.2}{\pi^{2}}\right) T^{3} .
$$

We consider the case where the $S$ scalar density is very small compared with the equilibrium density and solve the Boltzmann equation with $f=0$ on the right-hand side,

$$
\frac{d f}{d T}=-\frac{\left\langle\sigma_{\mathrm{ann}} v_{\mathrm{rel}}\right\rangle}{K} f_{0}^{2} .
$$

We take the Universe to be initially at a high temperature, $T \gg m_{W}$, and calculate the resulting relic density of $S$ scalars as the Universe cools. The annihilation cross sections of relativistic $S S^{\dagger}$ pairs to $t$ quarks, $W$ and $Z$ bosons, and the $h^{0}$ Higgs scalars (lighter quarks and leptons do not contribute significantly due to their very small Yukawa couplings) are estimated by using the center-of-mass annihilation cross sections calculated for $S$ scalars with typical energy $E_{T} \approx T$. We will see that the core results of the paper are not very sensitive to uncertainties in the calculation of the annihilation cross section and thermal relic $S$ density. The relativistic annihilation cross sections $\sigma_{i}$ may be obtained from the nonrelativistic $\left\langle\sigma_{\mathrm{ann}} v_{\text {rel }}\right\rangle$ given in [20] via the relation $\sigma_{i}=(1 / 2)\left\langle\sigma_{\text {ann }} v_{\text {rel }}\right\rangle\left(m_{S} \rightarrow E_{T}\right)$ (where $i \equiv t, W, Z, h^{0}$ denotes the standard model particle in question), which may be confirmed by directly calculating the cross sections. For $T<m_{i}$ the contribution of $\sigma_{i}$ to the total cross section is zero, which models Boltzmann suppression. Then $\left\langle\sigma_{i} v_{\text {rel }}\right\rangle=2 \sigma_{i}$, where we take $v_{\text {rel }}=2$ for relativistic annihilations [29]. For relativistic $S$ scalars, $f_{0}=1.2 / \pi^{2}$ is a constant so Eq. (10) can be integrated as

$$
f_{i}=-2 f_{0}^{2} \int_{E_{T_{0}}}^{E_{T_{f}}} d E_{T} \frac{\sigma_{i}}{K},
$$

where $E_{T}=T, E_{T_{f}}=m_{i}$, and the initial thermal energy $E_{T_{0}} \rightarrow \infty$. We will take $K \propto g(T)^{1 / 2}$ to be constant with $g(T)=g\left(T_{i}\right)$, where $T_{i}=m_{i}$, since most of the integral comes from $E_{T}$ close to $m_{i}$. The total contribution to $f$ is then 


$$
\begin{array}{r}
f_{T}=\sum f_{i}=1.3 \times 10^{12} \lambda_{S}^{2}\left[1+0.27\left(\frac{115 \mathrm{GeV}}{m_{h}}\right)\right. \\
\left.+0.20\left(\frac{m_{h}}{115 \mathrm{GeV}}\right)^{2}\right]
\end{array}
$$

In this we have taken $g\left(T_{i}\right)=106.75$, corresponding to the standard model degrees of freedom and $\lambda_{t}=0.7$ (corresponding to $m_{t}=175 \mathrm{GeV}$ ). In addition, in order to obtain an analytical result we have expanded the Higgs propagators in $\sigma_{i}(i=W, Z, t)$ assuming that $4 E_{T}^{2}$ is large compared with $m_{h}^{2}$, which is generally satisfied if $m_{h}^{2}$ is small compared with $4 m_{W}^{2}$. (We refer to this as the small Higgs mass limit.)

The $S$ number density from the decay of thermal equilibrium $h^{0}$ scalars at temperatures less than the electroweak phase transition (where $T_{\mathrm{EW}} \gtrsim 1.5 m_{h}$ [30]) is given by

$$
\frac{d n_{S}}{d t}+3 H n_{S}=\left\langle\Gamma_{h^{0}}\right\rangle n_{h^{0} \mathrm{eq}},
$$

where $H$ is the expansion rate and the thermal equilibrium density of $h^{0}, n_{h^{0} \mathrm{eq}}$, is given by

$$
n_{h^{0} \mathrm{eq}}=\frac{1}{2 \pi^{2}} \int_{m_{h}}^{\infty} \frac{E\left(E^{2}-m_{h}^{2}\right)^{1 / 2}}{\left(e^{E / T}-1\right)} d E,
$$

and the decay rate for $h^{0}$ scalars with energy $E$ is

$$
\Gamma_{h^{0}}=\frac{\lambda_{S}^{2} v^{2}}{16 \pi E}
$$

Thus the thermal average of the decay rate is

$$
\begin{gathered}
\left\langle\Gamma_{h^{0}}\right\rangle=\frac{1}{n_{h^{0} \mathrm{eq}}} \frac{\lambda_{S}^{2} v^{2} T^{2} e^{-m_{h} / T} \eta\left(m_{h} / T\right)}{32 \pi^{3}} ; \\
\eta(a)=\int_{0}^{\infty} \frac{t^{1 / 2}(t+2 a)^{1 / 2}}{\left(e^{t}-e^{-a}\right)} d t .
\end{gathered}
$$

Therefore in terms of $f$, the $S$ density from $h^{0}$ decays is given by

$\frac{d f}{d T}=-\frac{\left\langle\Gamma_{h^{0}}\right\rangle f_{h^{0} \mathrm{eq}}}{K T^{3}} \equiv-\frac{\eta\left(m_{h} / T\right)}{K T^{4}} \frac{\lambda_{S}^{2} v^{2} e^{-m_{h} / T}}{32 \pi^{3}}$.

$\eta(a)$ is a slowly varying function of $a$, with $\eta(0)=1.64$, $\eta(1)=1.87$, and $\eta(5)=3.00$. Since most of the contribution to $f$ comes from $m_{h} / T \sim 1$, we take $\eta\left(m_{h} / T\right)$ to be equal to $\eta(1)$, in order to obtain an analytical expression. Therefore the density of $S$ scalars from $h^{0}$ decay, $f_{\text {dec }}$, is given by

$f_{\text {dec }}=\frac{\lambda_{S}^{2} v^{2} \eta(1)}{16 \pi^{3} K m_{h}^{3}} \simeq 1.08 \times 10^{14} \lambda_{S}^{2}\left(\frac{115 \mathrm{GeV}}{m_{h}}\right)^{3}$.

In this we have assumed that $v$ is given by its $T=0$ value, $v=250 \mathrm{GeV}$. Since most of the contribution to $f_{\mathrm{dec}}$ comes from $T \lesssim m_{h}<T_{\mathrm{EW}}$, this should be a reasonable approximation. We see that the $S$ density from $h^{0}$ decays is generally much larger than that from $2 \leftrightarrow 2$ annihilation processes in the small Higgs mass limit. For larger values of the Higgs mass, it is possible that $s$-channel pole annihilations [29] may result in $2 \leftrightarrow 2$ processes dominating the $h^{0}$ decays [31], in which case $f_{\text {dec }}$ is a lower bound on the number of $S$ scalars produced thermally.

The resulting density of $S$ plus $S^{\dagger}$ scalars is then the sum of scattering and decay contributions,

$$
\Omega_{S}=\frac{2 m_{S}}{\rho_{c}} g\left(T_{\gamma}\right) T_{\gamma}^{3} \sum \frac{f_{i}}{g\left(T_{i}\right)},
$$

where $\rho_{c}=7.5 \times 10^{-47} h^{2} \mathrm{GeV}^{4}$ is the critical density, $T_{\gamma}=2.4 \times 10^{-4} \mathrm{eV}$ is the present photon temperature, $T_{i} \approx m_{i}$, and $g\left(T_{\gamma}\right)=2$. In this we have used the fact that the $S$ number density to entropy is conserved once the scattering and decay processes are Boltzmann suppressed, such that $g(T) n_{S} / T^{3}$ is constant. Therefore with $f \approx$ $f_{\text {dec }}$ and $g\left(T_{i}\right)=106.75$ for $T_{i} \approx m_{h}$, the thermal relic $S$ density $\Omega_{S}$ is related to $\lambda_{S}$ by

$$
\begin{aligned}
\lambda_{S}= & 2.0 \times 10^{-10} \frac{h}{\eta^{1 / 3}}\left(\frac{\Omega_{S}}{0.3}\right)^{1 / 2} \\
& \times\left(\frac{10 \eta^{2 / 3} \mathrm{MeV}}{m_{S}}\right)^{1 / 2}\left(\frac{m_{h}}{115 \mathrm{GeV}}\right)^{3 / 2} .
\end{aligned}
$$

Thus, for Higgs masses in the range $115 \mathrm{GeV}$ to $1 \mathrm{TeV}$ and with expansion rate $h \approx 0.7$, the upper bound on $\lambda_{S}$ from requiring that $\Omega_{S} \lesssim 0.3$ is in the range $(1.4 \times$ $\left.10^{-10}-3.6 \times 10^{-9}\right) \eta^{-1 / 3}$. This is in broad agreement with the upper bound estimated in [24], based on the weaker condition that the $S$ scalars do not come into thermal equilibrium. More importantly, we see that it is possible to generate a thermal relic density with $\Omega_{S} \approx 0.3$ and $m_{S} \approx 10 \mathrm{MeV}$ (typical of SIDM scalars) purely within the minimal gauge singlet scalar extension of the standard model.

4. Naturally consistent thermal relic SIDM for zero bare mass. - The value of $\lambda_{S}$ from requiring that $\Omega_{S} \approx 0.3$ is satisfied is not very much smaller than the upper limit Eq. (7) from the requirement that the Higgs expectation value contribution to the $S$ mass is compatible with perturbative SIDM $S$ scalars. This suggests that it is quite likely that all the $S$ mass might come from its interaction with the Higgs scalar when its relic density is sufficient to account for dark matter. If we assume that all the $S$ mass is due to the Higgs expectation value, then we find that the $S$ mass is fixed by the thermal relic density

$$
m_{S}=2.9\left(\frac{\Omega_{S}}{0.3}\right)^{1 / 5}\left(\frac{h}{0.7}\right)^{2 / 5}\left(\frac{m_{h}}{115 \mathrm{GeV}}\right)^{3 / 5} \mathrm{MeV}
$$

We refer to this as the thermal relic $S$ mass. Comparing with the Spergel-Steinhardt range for SIDM,

$$
m_{S}=(6.6-15.4) \eta^{2 / 3} \mathrm{MeV},
$$

we see that the thermal relic mass for $S$ scalars is within the range required to account for SIDM when the self-coupling constant $\eta$ is equal to about 0.1 , a natural value which is consistent with the Higgs doublet self-coupling in the standard model. The thermal relic mass is not strongly dependent upon cosmological parameters, nor is it strongly 
dependent upon the Higgs mass. In particular, it is relatively insensitive to uncertainties in the calculation of $f$, since a change in $f$ by a factor $\delta$ produces a change in $\Omega_{S}$ by the same factor, and so a change in the thermal relic mass by $\delta^{1 / 5}$. The coupling corresponding to the thermal relic mass is

$$
\lambda_{S}=2.7 \times 10^{-10}\left(\frac{\Omega_{S}}{0.3}\right)^{2 / 5}\left(\frac{h}{0.7}\right)^{4 / 5}\left(\frac{m_{h}}{115 \mathrm{GeV}}\right)^{6 / 5} .
$$

This suggests a scenario for dark matter in which stable gauge singlet scalars couple very weakly to the standard model sector but self-couple with a relatively strong coupling of about 0.1 , of the order expected from the example of the standard model Higgs self-coupling.

5. Conclusions.-We have considered the thermal generation of a relic density of SIDM gauge singlet scalars. The dominant process for small Higgs mass is the decay of thermal equilibrium Higgs scalars to gauge singlet scalar pairs. For SIDM scalars with perturbative self-interactions, the mass must be no greater than around $30 \mathrm{MeV}$. For such light scalars, the requirement of an acceptable relic density of $S$ scalars requires that the $S$ coupling to the standard model Higgs satisfies $\lambda_{S} \lesssim 10^{-(9-10)}$. This limit comes from the requirement that $S$ scalars are not thermally overproduced, which is a stronger condition than requiring that they do not come into thermal equilibrium.

In the case where $S$ scalars account for dark matter and where the $S$ mass is entirely due to the Higgs expectation value, we find that the $S$ mass is fixed by the thermal relic dark matter density to be between about 2.9 and $10.5 \mathrm{MeV}$ for Higgs masses ranging from $115 \mathrm{GeV}$ to $1 \mathrm{TeV}$. (The upper limit on the $S$ mass may be smaller if $2 \leftrightarrow 2$ annihilations dominate $h^{0}$ decays for large Higgs mass.) This is very similar to the range of masses $\left[(6.6-15.4) \eta^{2 / 3} \mathrm{MeV}\right]$ required by self-interacting dark matter with self-coupling $\eta$ of the order of the natural value (based on comparison with the standard model Higgs doublet self-coupling) of around 0.1 . This result is not strongly sensitive to uncertainties either in the cosmological parameters or in the calculation of the thermal relic $S$ density. We find this coincidence remarkable and a possible hint that light gauge singlet scalars with very weak coupling to the standard model sector may play an important role in cosmology and particle physics.

The author thanks the CERN theory division for its hospitality and PPARC for a Travel Fund award.

\footnotetext{
*Present address: Theoretical Physics Division, Department of Mathematical Sciences, University of Liverpool, Liverpool L69 3BX, UK.

Electronic address: mcdonald@sune.amtp.liv.ac.uk
}

[1] J. F. Navarro, C. S. Frenk, and S. D. M. White, Astrophys. J. 462, 563 (1996); S. Ghigna, B. Moore, F. Governato, G. Lake, T. Quinn, and J. Stadel, ibid. 544, 616 (2000).

[2] B. Moore, T. Quinn, F. Governato, J. Stadel, and G. Lake, Mon. Not. R. Astron. Soc. 310, 1147 (1999).

[3] B. Moore, S. Ghigna, F. Governato, G. Lake, T. Quinn, J. Stadel, and P. Tozzi, Astrophys. J. 524, L19 (1999).

[4] A. Klypin, A. V. Kravtsov, O. Valenzuela, and F. Prada, Astrophys. J. 522, 82 (1999).

[5] C. Firmani, E. D’Onghia, V. Avila-Reese, G. Chincarini, and X. Hernandes, Mon. Not. R. Astron. Soc. 315, L29 (2000).

[6] J. F. Navarro and M. Steinmetz, Astrophys. J. 528, 607 (2000).

[7] H. J. Mo and S. Mao, astro-ph/0002451.

[8] V. P. de Battista and J. A. Sellwood, Astrophys. J. 493, L5 (1998).

[9] R. A. Swaters, B. F. Madore, and M. Trewhella, Astrophys. J. 531, L107 (2000); F. C. van den Bosch, B. E. Robertson, J. J. Dalcanton, and W. J. G. de Blok, Astrophys. J. 119, 1579 (2000).

[10] J. S. Bullock, A. V. Kravtsov, and D. H. Weinberg, Astrophys. J. 539, 517 (2000).

[11] R. Dave, D. N. Spergel, P. J. Steinhardt, and B. D. Wandelt, Astrophys. J. 547, 574 (2001).

[12] D. N. Spergel and P. J. Steinhardt, Phys. Rev. Lett. 84, 3760 (2000).

[13] B. D. Wandelt, R. Dave, G. R. Farrar, P. C. McGuire, D. N. Spergel, and P. J. Steinhardt, astro-ph/0006344.

[14] C. Firmani, E. D’Onghia, and G. Chincarini, astro-ph/ 0010497; N. Yoshida, V. Springel, S. D. M. White, and G. Tormen, Astrophys. J. 535, L103 (2000); 544, L87 (2000); A. Burkert, ibid. 534, L143 (2000); C. S. Kochanek and M. White, ibid. 543, 514 (2000).

[15] J. Miralda-Escude, astro-ph/0002050.

[16] J. S. B. Wyithe, E. L. Turner, and D. N. Spergel, Astrophys. J. 555, 504 (2001).

[17] J. A. Tyson, G. P. Kochanski, and I. P. Dell'antonio, Astrophys. J. 498, L107 (1998).

[18] J. P. Ostriker, Phys. Rev. Lett. 84, 5258 (2000).

[19] V. Silveira and A. Zee, Phys. Lett. 161B, 136 (1985).

[20] J. McDonald, Phys. Rev. D 50, 3637 (1994).

[21] C. Burgess, M. Pospelov, and T. ter Veldhuis, hep-ph/ 0011335 .

[22] B. W. Lee and S. Weinberg, Phys. Rev. Lett. 39, 165 (1977).

[23] M. C. Bento, O. Bertolami, R. Rosenfeld, and L. Teodoro, Phys. Rev. D 62, 041302(R) (2000).

[24] M. C. Bento, O. Bertolami, and R. Rosenfeld, Phys. Lett. B 518, 276 (2000).

[25] D. E. Holz and A. Zee, Phys. Lett. B 517, 239 (2001).

[26] The ALEPH Collaboration, R. Barate et al., Phys. Lett. B 495, 1 (2000); The L3 Collaboration, M. Acciarri et al., Phys. Lett. B 495, 18 (2000).

[27] LEP Working Group, Report No. CERN-EP/2001-021.

[28] J. R. Forshaw, D. A. Ross, and B.E. White, hep-ph/ 0107232.

[29] P. Gondolo and G. Gelmini, Nucl. Phys. B360, 145 (1991).

[30] K. Jansen, hep-lat/9509018.

[31] J. McDonald (to be published). 